\title{
ADDITIONAL PULMONARY VALVE CLOSURE CAUSED BY THE CATHETER AND DEMONSTRATED BY THE INTRACARDIAC PHONOCARDIOGRAM
}

\author{
BY \\ LEON RESNEKOV AND JANE SOMERVILLE \\ From the Institute of Cardiology and National Heart Hospital, London
}

Received August 7, 1963

Simultaneous recording of intracardiac pressures and heart sounds has been made possible by the introduction of a high-frequency micromanometer developed by Soulié et al. (1954). Using this technique Soulié et al. (1961) have reported their findings in congenital and acquired heart disease. Analysing the second heart sound in recordings from the pulmonary artery and right ventricle in 11 cases of atrial septal defect, they report that splitting of the second sound does not appear to be synchronous with what has always been presumed to be aortic and pulmonary valve closure. On this basis conventional theories about recording the second heart sound in atrial septal defect were challenged.

While investigating the value of intracardiac phonocardiography we have particularly studied that sound which has been conventionally called the pulmonary second sound (P2) and thought to correspond to pulmonary valve closure. In addition a sound following P2 has frequently been recorded from the main pulmonary artery and this has been called P3 (see Fig. 1). When sounds and pressures are recorded with the catheter tip high in the outflow tract of the right ventricle another extra sound which has been named RVP3 (see Fig. 2) has been seen to follow conventional closure of the pulmonary valve (P2). In order to elucidate the mechanism of these additional sounds we have studied their incidence and characteristics and correlated the findings on the intracardiac phonocardiogram with the hæmodynamic changes present in each case.

\section{METHOD}

Using the Allard-Laurens micromanometer mounted at the end of a twin-lumen $8 \mathrm{~F}$ cardiac catheter, pressure and sound records have been simultaneously recorded from the right side of the heart in 30 unselected patients with congenital or acquired heart disease (Table I). The natural frequency of the micromanometer is around 2300 c.p.s. and linearity is better than \pm 2 per cent from $0-300 \mathrm{~mm}$. $\mathrm{Hg}$. The electrocardiogram and respiration, and an external phonocardiogram, using a conventional microphone on the left sternal edge, were also recorded. An indirect carotid artery tracing was obtained using a carbon granule microphone in order to fix closure of the aortic valve (A2). All records were taken simultaneously at a paper speed of $80 \mathrm{~mm}$./ $\mathrm{sec}$. using a NEP 6-channel photographic recorder. Routine pressure measurements were made and pulmonary and systemic flows calculated by the Fick principle (1870). Pulmonary valve closure (P2) was identified as the sound occurring synchronously with the dicrotic notch on the pulmonary artery tracing, and $\mathrm{A} 2$ as the sound oocurring just before the incisura on the indirect carotid artery tracing allowing 0.02-0.04 sec. delay in transmission of aortic valve events. The intracardiac phonocardiogram was recorded in the main pulmonary artery and its branches, the body and outflow tract of the right ventricle, right atrium, and superior vena cava. Each record was carefully scrutinized for additional sounds following P2. The additional sounds recorded in the pulmonary artery and right ventricular outflow 


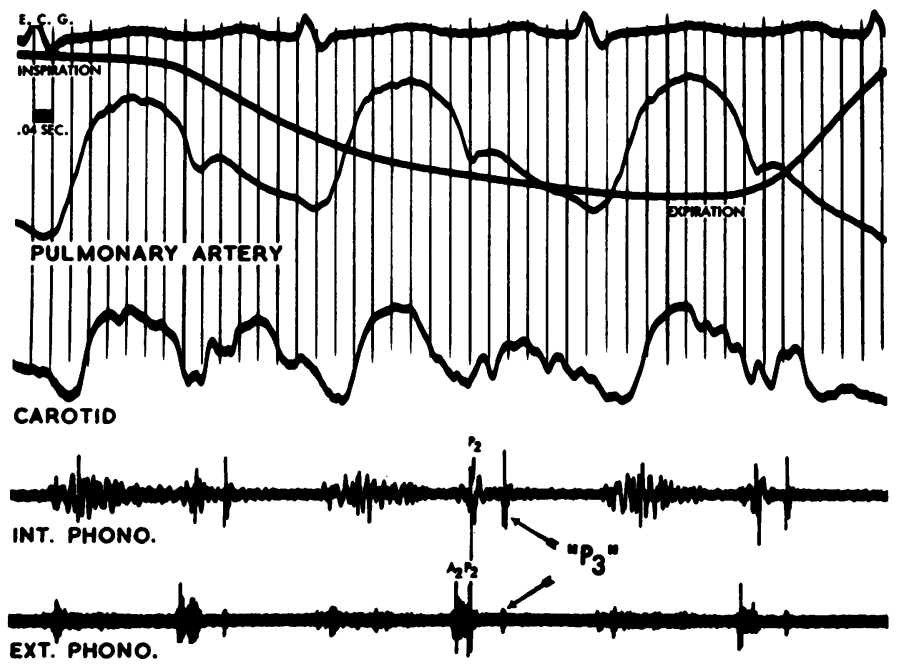

FIG. 1.-P3 recorded in the pulmonary artery from a patient with an uncomplicated atrial septal defect. From above downwards are shown: E.C.G., lead II; respiration; pulmonary artery: pressure pulse from the main pulmonary artery recorded by the micromanometer; carotid: indirect carotid artery tracing using a carbon granule microphone; Int. Phono: internal phonocardiogram recorded from within the main pulmonary artery by the micromanometer; Ext. Phono: conventional phonocardiogram recorded from the lower sternal edge using a piezo-crystal microphone. Paper speed: $80 \mathrm{~mm}$./sec. An ejection systolic murmur is present within the pulmonary artery. Aortic valve closure (A2) is recorded only on the external phonocardiogram. Pulmonary valve closure (P2) is synchronous with the dicrotic notch of the pulmonary artery pressure pulse and is recorded with no delay on the internal and external phonocardiogram. An additional sound (P3) follows P2 and is recorded both internally and externally. Note the slight notch on the pressure record corresponding to P3. The second heart sound (A2 P2) does not move with respiration.

tract were called P3 and RVP3 respectively. The P2-P3 interval range was measured and it was also noted whether a diastolic murmur followed in the P2-RVP3 interval. In 3 of the 30 cases the external phonocardiogram was recorded with a conventional $8 \mathrm{~F}$ cardiac catheter in the pulmonary artery, right atrium, and superior vena cava and the records examined for additional diastolic sounds.

TABLE I

Diagnosis of The Patients Studied (age range 8-57 years)

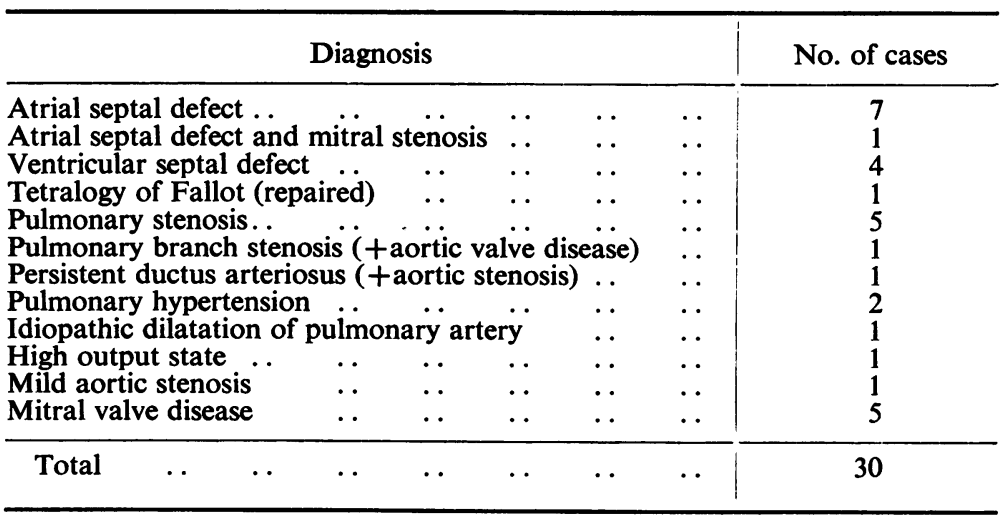




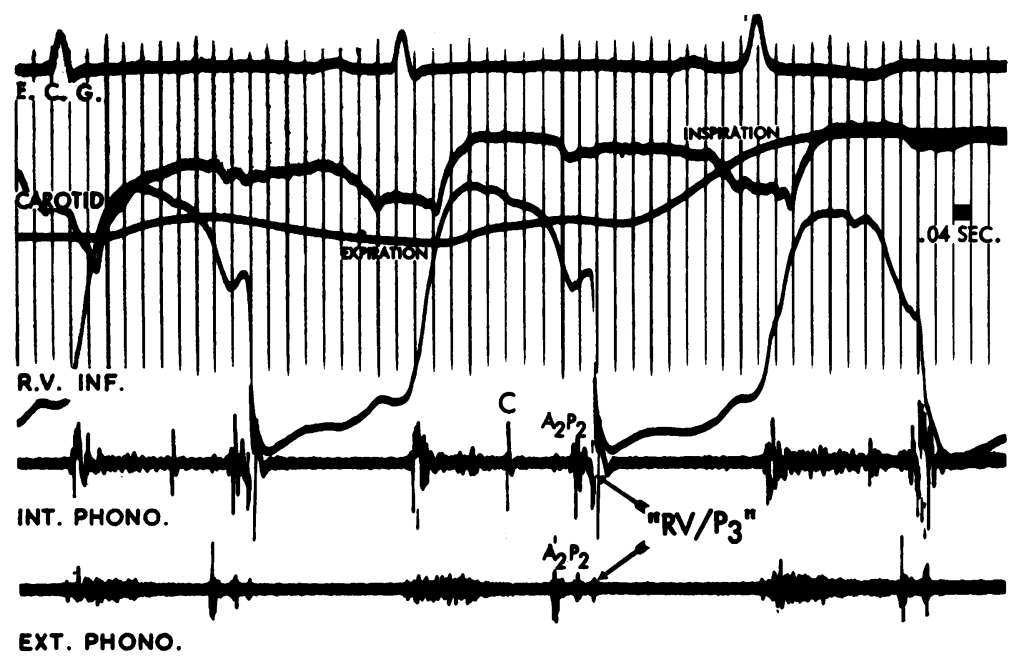

FIG. 2.-RVP3 recorded from the outflow tract of the right ventricle in a patient with an uncomplicated atrial septal defect. From above downwards are shown: E.C.G.: lead II; carotid: indirect carotid artery tracing using a carbon granule microphone; respiration; R.V. Inf.: pressure pulse recorded in the outflow tract of the right ventricle by the micromanometer; Int. Phono.: internal phonocardiogram recorded by the micromanometer at this site; Ext. Phono.: conventional phonocardiogram recorded at the left sternal edge using a piezo-crystal microphone; paper speed: $80 \mathrm{~mm}$./sec. A systolic murmur is recorded internally and externally. Aortic valve closure (A2) is well shown on the external phonocardiogram and is only just recorded on the internal sound channel. Pulmonary valve closure (P2) is clearly recorded on both sound channels. The micromanometer lies in the outflow tract of the right ventricle at the beginning of the cardiac cycle. With each systolic contraction the tip of the catheter enters the pulmonary artery marked by a sound $C$ on the internal phonocardiogram. Pulmonary valve closure (P2) now occurs with the catheter tip still in the pulmonary artery. The tip is then drawn back into the outflow tract of the right ventricle (note acute pressure drop), the pulmonary valve shuts again completely, giving rise to RVP3 recorded internally and externally. There is variation in the width of the characteristically deformed right ventricular pressure pulse corresponding to variation in the P2-RVP3 interval. A diastolic murmur can also be seen between P2 and RVP3 (see text).

\section{RESULTS}

The findings are shown in Table II. P3 was recorded in 24 patients. In the 6 patients where P3 was not recorded (Cases 6, 7, 9, 17, 19,21), pulmonary stenosis was present, which was at valvar level in 5 and at infundibular level in 1. In these 6 patients P2 was delayed and diminished in intensity. Mild infundibular pulmonary stenosis was present in one patient in whom P3 was recorded (Case 27), but P2 in this case was not diminished. P3 was intense $(+++)$ in the main pulmonary artery in 6 patients (Cases $3,10,14,15,23,24)$ and moderately intense $(++)$ in a further 6 (Cases 1, 8, 12, 13, 25, 30). In all these patients the sound was also recorded on the external phonocardiogram. The intensity of P3 always diminished when the catheter was in a branch of the pulmonary artery as compared with the main pulmonary artery (Fig. 3). Of the patients with intense or moderately intense $\mathrm{P} 3,11$ had either increased pulmonary artery pressure or increased pulmonary blood flow due to a left-to-right shunt at atrial or ventricular level. Case 30 in whom P2 and P3 were extremely loud had a high output state in the absence of valvular heart disease or intracardiac shunt. In 23 of the patients where P3 was clearly recorded the P2-P3 interval was measured. In one patient (Case 5) it was not possible to designate clearly P3 in all cycles owing to an intense murmur recorded in the pulmonary artery. The P2-P3 interval was longest where the output of the right ventricle was high and the right ventricle active, as in atrial septal defect and other left-to-right shunts. Usually the larger the left-to-right shunt, the wider was the P2-P3 interval. Where P3 was recorded but the P2-P3 interval short, output of the right ventricle was generally low. This was particularly shown in the patients with complicated mitral valve disease (see Table II).

RVP3 was recorded in 25 cases with the catheter tip positioned in the outflow tract of the right ventricle just below the pulmonary valve. Pulmonary valve stenosis was present in the five patients 
TABLE II

Hamodynamic Findings and Characteristics of P3 and RVP3 in the 30 Patients Studied

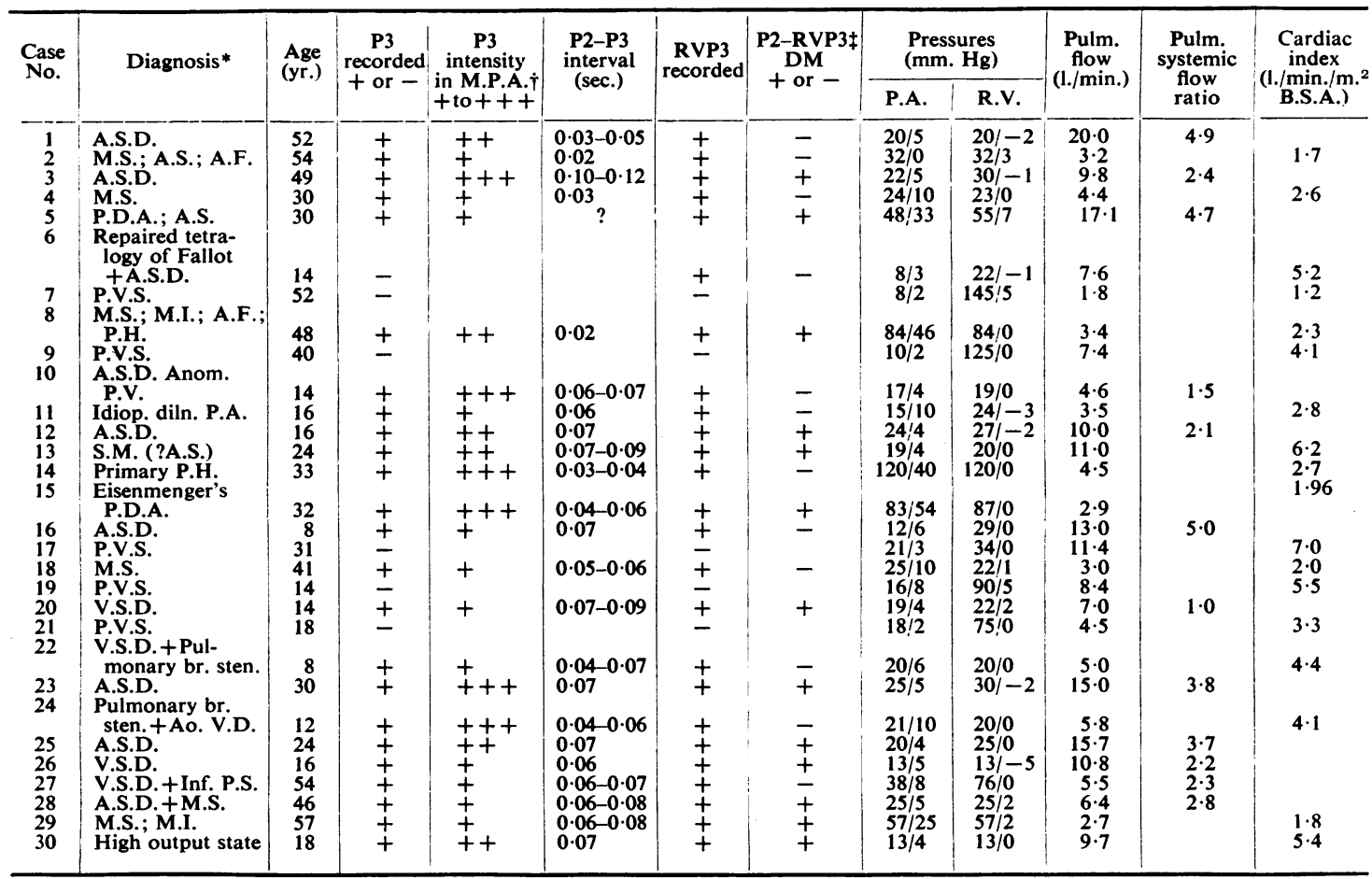

* Anom. P.V.= anomalous pulmonary venous drainage; A.F.=atrial fibrillation; Ao.V.D.=aortic valve disease; A.S.=aortic stenosis; A.S.D. = atrial septal defect; Idiop. diln. P.A. =idiopathic dilatation of the pulmonary artery; Inf. P.S.=infundibular pulmonary stenosis M.I.=mitral incompetence; M.S. = mitral stenosis; P.D.A=persistent ductus arteriosus; P.H=pulmonary hypertension; Pulmonary br. sten.= pulmonary branch stenosis; P.V.S.=pulmonary valve stenosis; S.M.=systolic murmur; V.S.D.=ventricular septal defect

sten $=$ pulmonary branch stenosis; $\mathbf{P}$.

$\ddagger$ P2-RVP3 DM = diastolic murmur in the interval between P2 and RVP3.

E. C. G.
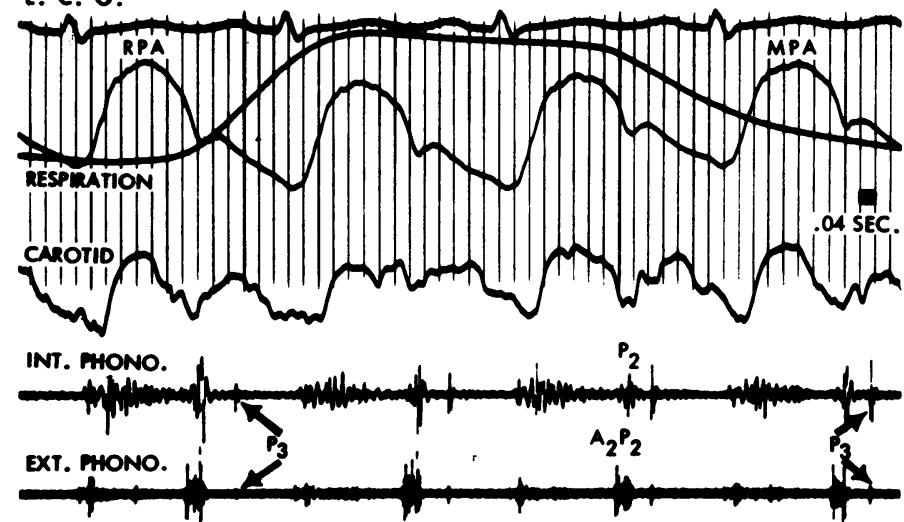

FIG. 3.-P3 becoming louder as the catheter tip approaches the pulmonary valve. The layout is as before, and paper speed $80 \mathrm{~mm}$. $/ \mathrm{sec}$. On the left the micromanometer is recording from within the right branch of the pulmonary artery (RPA). P3 is shown on the internal and external phonocardiograms. The catheter is being withdrawn to the main pulmonary artery (MPA), and P3 becomes louder as the pulmonary valve is approached. 


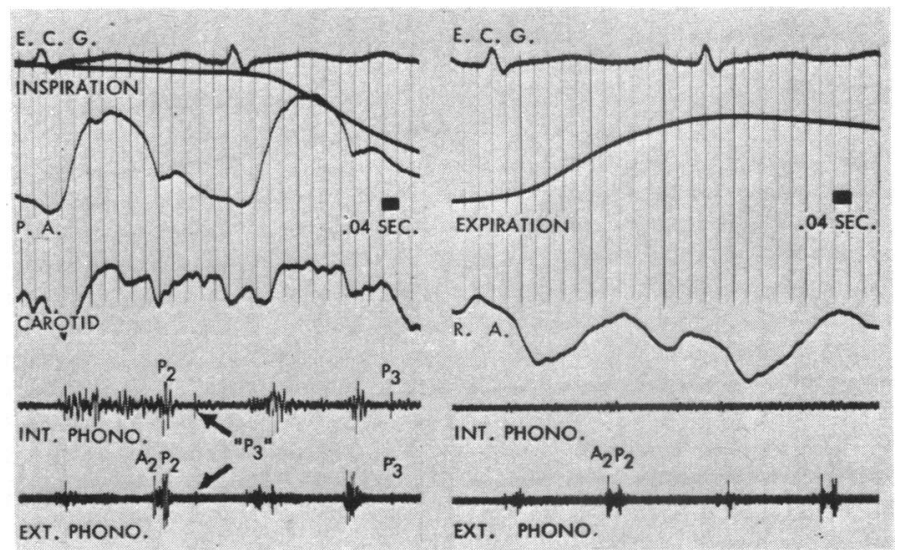

FIG. 4.-P3 recorded with the micromanometer catheter from within the pulmonary artery is absent in records from the right atrium. On the left the micromanometer records pressure and sound simultaneously from within the pulmonary artery. P3 is seen on both the internal and external phonocardiograms. On the right the catheter has been withdrawn to the right atrium and P3 has not been recorded.

(Cases 7, 9, 17, 19, 21) where RVP3 was not recorded. The obstruction was moderate or severe in all but one patient (Case 17). A diastolic murmur in the P2-RVP3 interval presumably due to pulmonary incompetence was recorded in 13 cases. P3 and RVP3 were never recorded by the intracardiac phonocardiogram in tracings from the body of the right ventricle, right atrium, or superior vena cava (Fig. 4).

In the three cases where the external phonocardiogram was recorded with a conventional catheter in the chambers of the right side of the heart, additional sounds with the same timing as P3 and RVP3 appeared when the catheter was in the pulmonary artery (Fig. 5) and outflow tract of the right

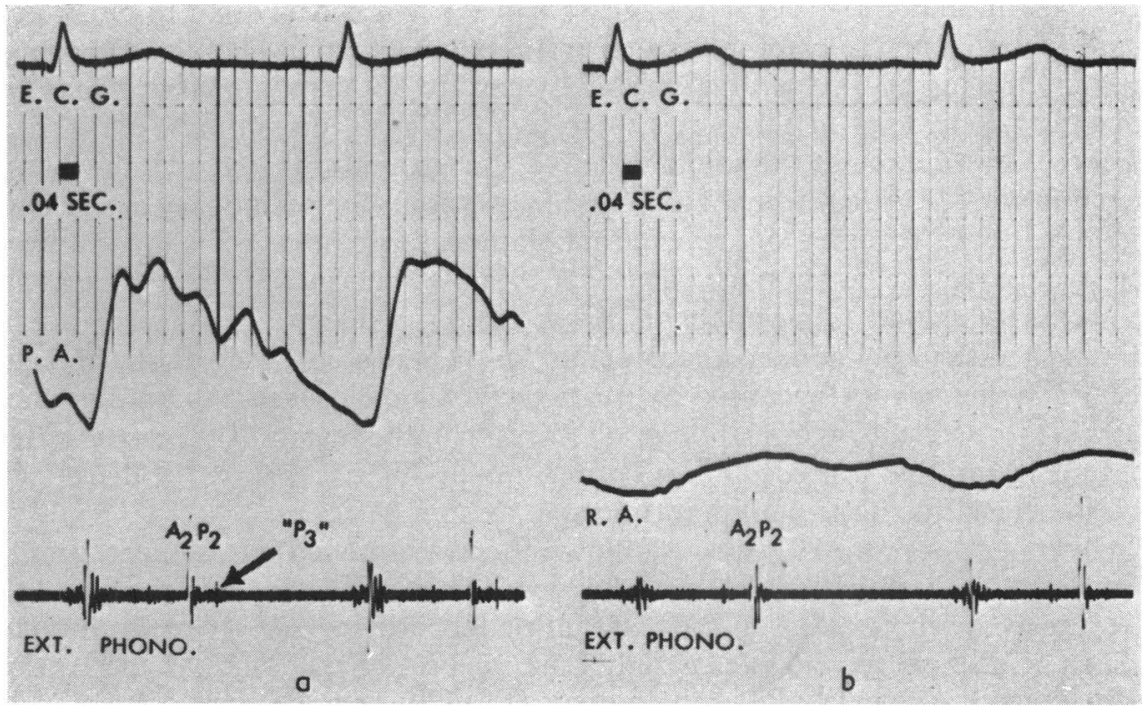

FIG. 5.-P3 produced by a conventional cardiac catheter. In (a) the catheter is in the main pulmonary artery and the pressure pulse (P.A.) is recorded using a Sanborn inductance manometer. The external phonocardiogram from the left sternal edge shows P3 following a closely split second sound (A2P2). A late systolic click can also be seen. In (b) the catheter has been withdrawn to the right atrium. P3 has not been recorded on the external phonocardiogram. 

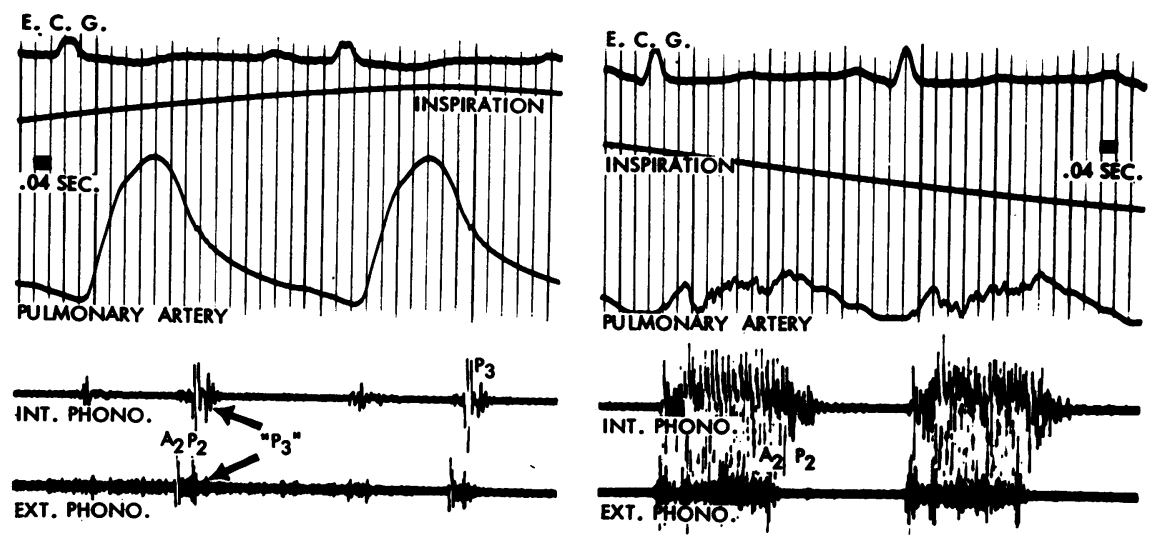

EXT. PHONO.

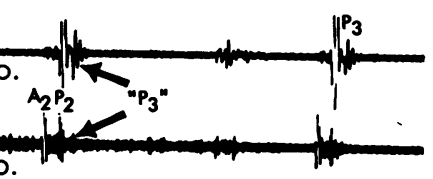

FIG. 6.-Variation in intensity of P3 with pulmonary artery pressure. On the left, P3 is shown on both the internal and external phonocardiogram recorded with the micromanometer in the main pulmonary artery of a patient whose pulmonary artery pressure was at systemic level. On the right, the micromanometer records from within the pulmonary artery in a case of severe valvar pulmonary stenosis. Note the intense ejection systolic murmur in the pulmonary artery and the delayed diminished pulmonary valve closure. P3 has not been recorded.

ventricle. The sounds were not present on the external phonocardiogram when the catheter tip lay in the body of the right ventricle, right atrium, or superior vena cava.

\section{Discussion}

The nature of the additional sounds P3 and RVP3 can only be understood when it is accepted that the sounds conventionally labelled $\mathrm{A} 2$ and $\mathrm{P} 2$ are produced by closure of the aortic and pulmonary valves. If $\mathrm{P} 2$ is caused by pulmonary valve closure it should correspond exactly with the dicrotic notch of the pulmonary artery tracing in a recording system with synchronous sound and pressure recording. Leatham and Gray (1956), using a system for which delay had to be allowed, assumed that the sound recorded on the external phonocardiogram which preceded the dicrotic notch on the pulmonary artery tracing by $0.02 \mathrm{sec}$. was caused by closure of the pulmonary valve (P2). With the micromanometer system used in this series there is no delay in pressure or sound recorded from within the heart. In all tracings the sound called P2 corresponds exactly with the dicrotic notch of the pulmonary artery. Furthermore P2 recorded from within the pulmonary artery is synchronous with the sound called P2 recorded on the external phonocardiogram. P2 is loudest when the tip of the catheter is close to the pulmonary valve. It is most intense when pulmonary valve closure would be expected to be loud as in pulmonary hypertension, and least intense when pulmonary valve closure is diminished as in pulmonary stenosis (Fig. 6). There seems little doubt, therefore, that the sound called P2 is unequivocally caused by pulmonary valve closure. As we have not made any direct measurements with the micromanometer recording in the region of the aortic valve it must be assumed that the sound that precedes $\mathrm{P} 2$ and occurs about $0.02 \mathrm{sec}$. before the dicrotic notch on the indirect carotid artery tracing is due to aortic valve closure (A2).

P3 is clearly separated from P2 which it follows after an interval that varies according to the hæmodynamic changes in each individual case and closely mimics the behaviour of P2. Like P2 it is frequently recorded synchronously on the external phonocardiogram. It varies in intensity in the different phases of respiration and is especially loud in cases of pulmonary hypertension. In contrast it may be difficult to identify $\mathrm{P} 3$ in the presence of pulmonary stenosis. It seems likely, therefore, that this additional diastolic sound is caused by the same mechanism as P2, namely, closure of the pulmonary valve. This concept is further supported by the fact that $\mathrm{P} 3$ is most intense when the catheter tip is near the pulmonary valve, and diminishes in intensity as the catheter tip passes away from the pulmonary valve into the pulmonary artery branches (Fig. 3). The sound P3 can only be 
recorded when the micromanometer catheter is through the pulmonary valve as shown in Fig. 4 . As this extra sound is not recorded in the region of the tricuspid valve it cannot be due to an opening snap of the tricuspid valve.

The production of $\mathrm{P} 3$ is not specific to the micromanometer catheter and can be seen on the external phonocardiogram with a conventional number $8 \mathrm{~F}$ catheter in the main pulmonary artery (see Fig. 5), but is not present with the catheter recording from other parts of the heart in the same case. Eldridge and Hultgren (1959) have also demonstrated additional sounds on the external phonocardiogram related to the presence of a conventional cardiac catheter through the pulmonary valve. It is possible that the sound they have called $\mathrm{X}$ is the same as $\mathrm{P}$.

The sound RVP3 recorded with the tip of the micromanometer catheter close to the pulmonary valve in the outflow tract of the right ventricle does not necessarily have the same time relation to P2 as does P3 in the same patient. However, it would appear that RVP3 results from a similar mechanism. When present it is always associated with a characteristically deformed pressure pulse which is produced by movement of the catheter tip in and out of the pulmonary valve. At the beginning of the cardiac cycle the catheter tip lies in the infundibulum of the right ventricle and as the systolic pressure rises the tip is forced into the pulmonary artery usually accompanied by a sound on the internal phonocardiogram labelled C (Fig. 2). The pulmonary valve closes at the expected time and $\mathbf{P} 2$ is recorded on the internal and external phonocardiogram. At this time the catheter tip is in the main pulmonary artery. When right ventricular diastole begins the catheter tip is drawn back into the right ventricle beneath the pulmonary valve. This is associated with a corresponding acute fall in pressure during which time the pulmonary valve now closes completely giving rise to RVP3 which is recorded simultaneously on the internal and external phonocardiogram. There is usually variation in the width of the deformed pressure pulse which corresponds with variation in the time interval between P2 and RVP3. We assume that RVP3 originates from the pulmonary valve for it is not recorded when the catheter tip is drawn away from the valve into the body of the RV where catheter movement cannot interfere with the closing mechanism of the pulmonary valve.

We have shown that P3 and RVP3 originate from the pulmonary valve, and their production depends on the presence of a catheter through this valve. Under these conditions pulmonary valve closure (P2) occurs at the expected time but is incomplete owing to the presence of the catheter. The catheter shaft is drawn back through the incompletely closed pulmonary valve during the most dynamic phase of right ventricular diastole, and it is likely that this movement mechanically causes the valve to open slightly. When the catheter stops moving with the tip remaining in the pulmonary artery, the pulmonary valve closes again, giving rise to P3. RVP3 recorded with the tip of the catheter in the infundibulum arises as a result of a similar mechanism, following the rapid withdrawal of the catheter tip in the pulmonary artery beneath the pulmonary valve. The P2-P3 interval varies with the stroke output and filling volume of the right ventricle and is therefore a measure of the most energetic phase of right ventricular diastole.

The recognition of these sounds and the understanding of their cause are important in the interpretation of intracardiac phonocardiography and for the study of the physiology of the heart in normal and abnormal states. P3 is easily identified as separate from P2 when records are taken at a sufficiently fast paper speed $(80 \mathrm{~mm}$./sec.). It is likely that these extra sounds were recorded by Soulié et al. (1961) who were unable to recognize their correct time and origin, as from published records it appears that their tracings were taken at too slow a paper speed. We are unable to agree that there is need to throw doubt on the conventional and accepted theories of the recording of the second heart sound. It is clear that the micromanometer catheter will produce and record additional sounds that must not be confused with normal physiological events.

\section{SUMMARY}

Intracardiac phonocardiographs were recorded from 30 unselected cases of congenital and acquired heart disease. Additional diastolic sounds have been recorded from the pulmonary 
artery (P3) and right ventricular infundibulum (RVP3). Both these sounds are related to the presence of the catheter through the pulmonary valve. It has been confirmed that the sound conventionally called $\mathbf{P} 2$ is caused by pulmonary valve closure.

The authors are grateful to the physicians of the National Heart Hospital who allowed their patients to be studied and to Mr. John Norman and Mr. John Manders for their technical help in obtaining the tracings.

\section{REFERENCES}

Eldridge, F. L., and Hultgren, H. N. (1959). Production of heart sounds by the cardiac catheter. Circulation, 19, 557.

Fick, A. (1870). Ueber die Messung des Blutquantums in den Herzventrikeln. S.-B. phys.-med. Ges. Würzb., 1870. [In Verh. phys.-med. Ges. Würzb. (1871). n.s., 2, heft 1 and 2, p. xvi].

Leatham, A., and Gray, A. (1956). Auscultatory and phonocardiographic signs of atrial septal defect. Brit. Heart J., $18,193$.

Soulié, C., Baculard, P., Bouchard, F., Cornu, Cl., Laurens, P., and Woolff, F. (1961). Le cathétérisme du cœur au micromanomètre. Le son intracardiaque. Arch. Mal. Caur, Suppl. 1.

Soulié, P., Laurens, P., Allard, E., and Bouchard, F. (1954). Le micromanomètre intracardiaque de Allard Laurens [résumé]. Acta cardiol. (Brux.), 9, 689. 